\title{
OXIDAÇÃO DO BORNEOL À CÂNFORA COM ÁGUA SANITÁRIA - UM EXPERIMENTO SIMPLES, DE BAIXO CUSTO E LIMPO
}

\author{
Ana Paula Bernardo dos Santos*, Idila Rafaela Carvalho Gonçalves, Karla Ceodaro Pais, Sabrina Teixeira Martinez, \\ Elizabeth Roditi Lachter e Angelo C. Pinto \\ Departamento de Química Orgânica, Instituto de Química, Universidade Federal do Rio de Janeiro, Cidade Universitária, Centro \\ de Tecnologia, Bl. A, 21949-900 Rio de Janeiro - RJ, Brasil
}

Recebido em 25/6/08; aceito em 25/2/09; publicado na web em 3/7/09

\begin{abstract}
OXIDATION OF BORNEOL TO CAMPHOR WITH BLEACH: A SIMPLE, GREEN CHEMISTRY AND INEXPENSIVE EXPERIMENT. Regulatory pressure is increasingly focusing on the use and disposal of substances hazardous to human health and environment. In the last years efforts have also been made to introduce green chemistry concepts in undergraduate courses. In this paper we present an experiment on the oxidation reaction of borneol to camphor with bleach in acetone. This experiment is important to show undergraduate students that a cheap and non hazardous commercial product can be a useful oxidation agent of alcohols.
\end{abstract}

Keywords: oxidation; borneol; bleach.

\section{INTRODUÇÃO}

A oxidação de álcoois aos seus derivados carbonílicos correspondentes é uma das transformações químicas mais comuns nas disciplinas experimentais de cursos de graduação de Química e áreas afins. ${ }^{1}$

Na literatura é descrito um grande número de reagentes para oxidação de álcoois. Muitos destes reagentes, principalmente aqueles à base de cromo, como $\mathrm{K}_{2} \mathrm{Cr}_{2} \mathrm{O}_{7} \mathrm{CrO}_{3}, \mathrm{PDC}$, PCC etc., apesar de muitas vezes proporcionarem bons rendimentos, vêm sendo substituídos por outros que não ofereçam riscos ao meio ambiente e à saúde humana. Outros reagentes, como $\mathrm{KMnO}_{4}$, são poucos seletivos, e alguns sais de rutênio, como $\mathrm{RuCl}_{3}$, são caros para serem utilizados em transformações químicas simples. ${ }^{2-5}$

Nos últimos anos, a preocupação com o meio ambiente é uma realidade e os conceitos de química limpa (química verde) vem ganhando cada vez mais força e adeptos nos Cursos de Química e áreas afins, com amplo apoio da sociedade. A química limpa tem como um de seus principais objetivos o uso de reagentes ou de processos que reduzam ou eliminem o uso e a geração de substâncias perigosas para o meio ambiente e a saúde humana. ${ }^{6-10}$

A água sanitária é um produto comercial obtido pela diluição de hipoclorito de sódio em água, que é estabilizado pela adição de cloreto de sódio. Este produto comercial possui um teor de cloro ativo em torno de $2,5 \%$, é alvejante e tem alto poder germicida, daí a razão porque é usado por donas de casa para a lavagem de roupas e para a desinfecção de muitos vegetais. ${ }^{11-13}$

"Cloro ativo" é o termo usado para definir espécies oxi-cloradas, tais como $\mathrm{HClO}$ e $\mathrm{ClO}^{-}$. Tais espécies estão presentes na água sanitária e apresentam ação microbicida, pois possuem a capacidade de se difundir através de membranas celulares e reagir com os aminoácidos que compõem as proteínas, alterando sua estrutura tridimensional e inativando-as. Este processo ocorre devido à dissociação em água do hipoclorito em ácido hipocloroso (Figura 1). Este ácido provoca o desenovelamento das proteínas, expondo seus aminoácidos e permitindo sua interação com aminoácidos de outras proteínas danificadas, aglutinando-as de forma irreversível. ${ }^{14}$ Além disto, o ácido hipocloroso também oxida grupos sulfidrilas de enzi-

*e-mail: anabernardo@iq.ufrj.br mas, inativando-as e provocando morte celular. ${ }^{14} \mathrm{O}$ ácido hipocloroso ( $\mathrm{HClO})$ é formado em nosso organismo como importante microbicida através da reação entre peróxido de hidrogênio e cloreto sob a ação de mieloperoxidases. ${ }^{15}$

$\mathrm{NaOCl}+\mathrm{CH}_{3} \mathrm{COOH} \longrightarrow \mathrm{HOCl}+\mathrm{CH}_{3} \mathrm{COONa}$

Figura 1. Reação de conversão de hipoclorito de sódio em ácido hipocloroso

O hipoclorito de sódio com $7 \%$ de cloro ativo, presente em produtos comerciais usados para desinfecção de piscinas tem sido usado para oxidação de alcoóis a cetonas, e na concentração de 5,8 ou $13 \%$, na presença de sais de índio, para cloração alílica. ${ }^{16-18}$

Nesta comunicação, é apresentada a oxidação de borneol à cânfora com água sanitária, um alvejante e desinfetante barato, disponível em estabelecimentos comerciais e supermercados, e largamente utilizado no nosso dia a dia, pelas sociedades de todo o mundo.

\section{PARTE EXPERIMENTAL}

\section{Oxidação do borneol à cânfora com água sanitária}

Num balão de fundo redondo de $100 \mathrm{~mL}$, colocou-se $1,0 \mathrm{~g}$ de borneol e, em seguida, adicionou-se $3,0 \mathrm{~mL}$ de acetona e $1,0 \mathrm{~mL}$ de ácido acético, sob agitação magnética, até completa solubilização do borneol. O balão foi colocado em banho-maria e acoplado a um condensador de refluxo. Após o banho-maria atingir $35^{\circ} \mathrm{C}$, $55 \mathrm{~mL}$ de água sanitária foram adicionados, gota a gota, pelo topo do condensador com auxílio de um funil de separação (ou funil de adição). Terminada a adição do agente oxidante, a mistura reacional foi mantida sob agitação por 45 min. Em seguida, fez-se a extração líquido-líquido, em funil de separação, com $15 \mathrm{~mL}$ de acetato de etila. A fase orgânica foi recolhida num Erlenmeyer de $150 \mathrm{~mL}$ e a fase aquosa retornada ao funil de separação para que a extração fosse repetida por mais 3 vezes, com $15 \mathrm{~mL}$ de AcOEt até a fase aquosa se tornar incolor.

A fase orgânica foi lavada com $40 \mathrm{~mL}$ de solução saturada de bicarbonato de sódio $\left(\mathrm{NaHCO}_{3}\right)$ e, posteriormente, com solução aquosa de bissulfito de sódio $5 \%(\mathrm{~m} / \mathrm{V})\left(\mathrm{NaHSO}_{3}\right)$. A fase orgânica foi secada com sulfato de magnésio $\left(\mathrm{MgSO}_{4}\right)$, filtrada e a solução concentrada à 
pressão reduzida em evaporador rotatório para a remoção do solvente, obtendo-se um sólido pastoso esbranquiçado. Este foi solubilizado em pequena quantidade de acetato de etila e transferido para um becher de $20 \mathrm{~mL}$, que foi mantido na capela até completa evaporação do solvente. Foi obtido um sólido branco de ponto de fusão $170{ }^{\circ} \mathrm{C}$ $\left(\mathrm{PF}_{\mathrm{Lit}}: 175-180{ }^{\circ} \mathrm{C}\right)$ com rendimento de $98 \%$.

\section{Determinação do teor de cloro ativo na água sanitária}

Para a determinação do teor de cloro ativo na água sanitária preparou-se uma solução de tiossulfato de sódio $0,1 \mathrm{~N}\left(\mathrm{Na}_{2} \mathrm{~S}_{2} \mathrm{O}_{3}\right.$ titulante), a qual foi padronizada com solução de iodato de potássio $0,1 \mathrm{~N}\left(\mathrm{KIO}_{3}\right)$. Após a padronização, o titulante foi transferido para uma bureta de $25 \mathrm{~mL}$.

Uma quantidade de $5 \mathrm{~mL}$ de água sanitária foi transferida com uma pipeta volumétrica para um balão volumétrico de $100 \mathrm{~mL}$, que foi completado com água. Uma alíquota de $10 \mathrm{~mL}$ desta solução foi transferida para um Erlenmeyer de $150 \mathrm{~mL}$, no qual foram colocados $30 \mathrm{~mL}$ de solução de iodeto de potássio $10 \%(\mathrm{~m} / \mathrm{V})$ e $30 \mathrm{~mL}$ de ácido acético.

Após este procedimento, procedeu-se à titulação com solução de tiossulfato de sódio $0,1 \mathrm{~N}$, até que a solução apresentasse uma cor amarela clara. Em seguida, adicionou-se 1,0 mL de solução de amido $5 \%(\mathrm{~m} / \mathrm{V})$ ao Erlenmeyer e continuou-se a titulação até que a cor azulada, que se formou após a adição da solução de amido, desaparecesse.

Foi medido o teor de cloro ativo em 3 amostras de águas sanitárias comerciais. Nestas 3 amostras foram consumidos $3,2 \mathrm{~mL}$ do titulante tiossulfato de sódio.

\section{Instrumentação e condições cromatográficas/Dados dos aparelhos}

Os espectros de infravermelho foram obtidos em espectrofotômetro de marca Nicolet, modelo Magna IR 760, utilizando pastilhas comprimidas de $\mathrm{KBr}$ anidro. Para confecção da pastilha foram utilizados $5 \mathrm{mg}$ da amostra. Os valores para a absorção foram referidos em número de onda, utilizando-se o centímetro recíproco $\left(\mathrm{cm}^{-1}\right)$ como unidade.

Para a obtenção dos espectros de $\mathrm{RMN}{ }^{1} \mathrm{H}$ e ${ }^{13} \mathrm{C}$ foram utilizados $30 \mathrm{mg}$ da amostra em $0,6 \mathrm{~mL}$ de $\mathrm{CDCl}_{3}$, à $25^{\circ} \mathrm{C}$, em tubos de $5 \mathrm{~mm}$ de diâmetro, a 200 e $50 \mathrm{MHz}$ para ${ }^{1} \mathrm{H} \mathrm{e}{ }^{13} \mathrm{C}$, respectivamente.

Os espectros de RMN foram obtidos em um espectrômetro Bruker, modelo Avance, operando a $200 \mathrm{MHz}$, e estão disponíveis no material suplementar.

As análises de CGAR-EM foram realizadas em cromatógrafo Agilent modelo 6890 acoplado a um detetor de massas Agilent modelo 5973, com ionização por impacto de elétrons a $70 \mathrm{eV}$ e analisador do tipo quadrupolo. A coluna capilar utilizada foi DB-1 HT (J\&W) $30 \mathrm{~m}$ x 0,25 $\mathrm{mm}$ x $0,10 \mu \mathrm{m}$ de espessura de fase. $O$ gás de arraste utilizado foi hélio $\left(0,8 \mathrm{~mL} \mathrm{~min}^{-1}\right)$ e a pressão na cabeça da coluna foi de 8 psi. A amostra foi injetada com volume de $0,1 \mu \mathrm{L}$ com divisão de fluxo de 20:1. A temperatura inicial do forno, injetor e detetor foi de 100, 270 e $280{ }^{\circ} \mathrm{C}$, respectivamente. A rampa empregada foi de $10{ }^{\circ} \mathrm{C}$ $\mathrm{min}^{-1}$, mantendo o aquecimento por 4,0 min na temperatura final de $250{ }^{\circ} \mathrm{C}$. O tempo total de corrida foi de 19,0 min e o cromatograma foi obtido no modo de varredura de íons totais (scan).

\section{RESULTADOS E DISCUSSÃO}

A utilização de hipoclorito de sódio para a oxidação de álcoois é bem documentada na literatura. ${ }^{17}$ Álcoois primários são oxidados a aldeídos ou ácidos carboxílicos e álcoois secundários a cetonas. Estas reações são realizadas, normalmente, na presença de ácidos fracos, levando à formação do ácido hipocloroso. Embora o mecanismo da reação não seja completamente compreendido, há evidências de que o intermediário hipoclorito de alquila seja formado, o qual leva à formação de um produto de eliminação via E2 como mostra a Figura $2 .{ }^{17}$
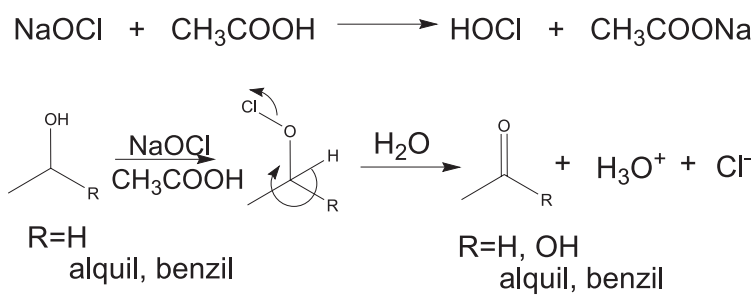

Figura 2. Reação de oxidação de álcoois primários e secundários com hipoclorito de sódio

Nesta comunicação optou-se pelo uso de água sanitária, cujo teor de cloro ativo foi de 2,5\%, ao invés de hipoclorito de sódio (10-13\%), porque a água sanitária pode ser adquirida em qualquer supermercado e em diferentes estabelecimentos comerciais.

O borneol foi escolhido porque a cânfora, seu produto de oxidação (Figura 3), é facilmente reconhecida pelo seu cheiro forte e penetrante e por ser muito usada em preparações farmacêuticas de uso tópico. A baixa toxidade $\left(\mathrm{LD}_{50}\right.$ em ratos: $3000 \mathrm{mg} / \mathrm{kg}$ ) destes dois monoterpenos permite que sejam manipulados em aulas experimentais sem maiores riscos. ${ }^{19}$
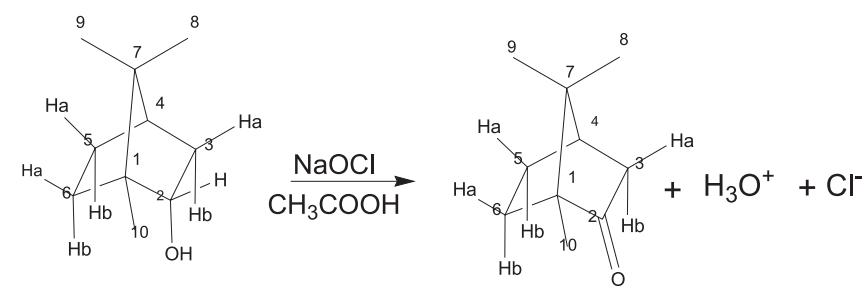

Figura 3. Reação de oxidação do borneol à cânfora com água sanitária

Antes de se proceder à reação de oxidação de borneol, avaliou-se o teor de cloro ativo em 3 amostras comerciais de água sanitária de diferentes produtores, adquiridas em supermercados, e em 4 amostras comercializadas em garrafas PET, em feiras livres de municípios do estado do Rio de Janeiro. As amostras de água sanitária apresentaram teor de cloro ativo em torno de $2,5 \%$, porém as amostras comercializadas em garrafas PET, devido à falta de regulamentação apresentaram teores de cloro ativo muito variados, estando fora das especificações da ANVISA.

A cânfora foi obtida com $98 \%$ de rendimento e $95 \%$ de conversão, sendo caracterizada pela espectroscopia de infravermelho (IV), de ressonância magnética nuclear (RMN) e cromatografia com fase gasosa acoplada à espectrometria de massas (CG/EM).

Os alunos poderão comprovar a obtenção da cânfora através da interpretação de seus espectros de infravermeIho, RMN de hidrogênio e carbono, e acompanhar a reação por cromatografia em fase gasosa ou camada fina. Outro aspecto importante do uso deste oxidante é a integração de um experimento clássico de Química Orgânica e de titulações volumétricas corriqueiras em disciplinas experimentais de Cursos Técnicos de Química, muito importantes para o controle de qualidade de produtos do cotidiano das donas de casa.

\section{CONCLUSÃO}

A água sanitária, um produto usado diariamente como alvejante de roupas e produzido no nosso organismo para a sua defesa con- 
tra patógenos invasores, pode ser usada como agente oxidante de álcoois em disciplinas experimentais de cursos de Química. Este é um bom exemplo de reação de oxidação que atende aos princípios de "Química Limpa" e que utiliza um reagente barato e acessível, oferecendo uma boa oportunidade para mostrar aos alunos de graduação como produtos presentes em seu cotidiano podem ser úteis em aulas experimentais.

\section{MATERIAL SUPLEMENTAR}

Está disponível em http://quimicanova.sbq.org.br, na forma de arquivo PDF, com acesso livre e apresenta os espectros de infravermelho, ressonância magnética nuclear de carbono e de hidrogênio, o cromatograma e os respectivos fragmentogramas da cânfora e borneol.

\section{AGRADECIMENTOS}

Á E. L. C. Oberosler (LADA-UFRJ), e ao Prof. D. S. Vaitsman (LADA-UFRJ) pelas sugestões e à CAPES e FAPERJ pelo apoio financeiro.

\section{REFERÊNCIAS}

1. De Luca, L.; Giacomelli, G.; Porcheddu, A.; J. Org. Chem. 2001, 66, 7907.

2. Chen, J. D.; Lempers, H. E. B.; Sheldon, R. A.; J. Chem. Soc., Faraday Trans. 1996, 92, 1807.

3. Corey, E. J.; Schmidt, G.;Tetrahedron Lett. 1979, 20, 399.
4. Gandhari, R.; Maddukuri, P. P.; Vinod, T. K.; J. Chem. Educ. 2007, 84, 852 .

5. Zhao, M.; Li, J.; Song, Z.; Desmond, R.; Tschaen, D. M.; Grabowski, E. J. J.; Reider, P. J.; Tetrahedron Lett. 1998, 39, 5323.

6. Trost, B. M.; Science 1991, 254, 1471

7. Dupont, J.; Quim. Nova 2000, 23, 825.

8. Bastos, R. S. B.; Cunha, A. S.; Silva, L. C.; Oliveira, C. C. P.; Rezende, C. M.; Pinto, A. C.; Quim. Nova 2008, 31, 172.

9. Manley, J. B.; Anastas, P. T.; Cue Jr., B. W.; J. Clean. Prod. 2008, 16, 743.

10. Lenardão, E. J.; Freitag, R. A.; Dabdoub, M. J.; Batista, A. C. F.; Silveira, C. C.; Quim. Nova 2003, 26, 123.

11. http://sbrtv1.ibict.br/upload/sbrt5485.pdf? PHPSESSID $=88$ b1b4d2cd3 443f5ba7c6b29362aed16, acessada em Junho 2009.

12. http://www.abipla.org.br/legisl/po89_94.htm, acessada em Junho 2008.

13. Thayer, A.; Chem. Eng. News 2007, 85 (25), 70.

14. Baird, C.; Química Ambiental, 2a . ed., Bookman: Porto Alegre, 2002.

15. Ohara, A.; Radicais Livres: bons, maus e naturais, Oficina de Textos, PróReitoria de Pesquisa da Universidade de São Paulo: São Paulo, 2006.

16. Stevens, R. V.; Chapman, K. T.; Weller, H. N.; J. Org. Chem. 1980, 45, 2030.

17. Pavia, D.; Lampman, G. M.; Kriz, G. S.; Engel, R. G.; Introduction to Organic Laboratory Techniques: A microscale Approach, $3^{\text {rd }}$ ed., Saunders College Publishing: Orlando, 1999, p. 266-278.

18. Pisoni, D. S.; Gamba, D.; Fonseca, C. V.; Costa, J. S.; Petzhold, C. L.; Oliveira, E. R.; Ceschi, M. A.; J. Braz. Chem. Soc. 2006, 17, 321.

19. Yoneda, J. D.; Leal, K. Z.; Seidl, P. R.; Azeredo, R. B.V.; Kleinpeter, E.; Quim. Nova 2007, 30, 2053. 


\section{OXIDAÇÃO DO BORNEOL À CÂNFORA COM ÁGUA SANITÁRIA - UM EXPERIMENTO SIMPLES, DE BAIXO}

\section{CUSTO E LIMPO}

Ana Paula Bernardo dos Santos*, Idila Rafaela Carvalho Gonçalves, Karla Ceodoro Pais, Sabrina Teixeira Martinez, Elizabeth Roditi Lachter e Angelo C. Pinto

Departamento de Química Orgânica, Instituto de Química, Universidade Federal do Rio de Janeiro, Cidade Universitária, Centro de Tecnologia, Bl. A, 21949-900 Rio de Janeiro - RJ, Brasil

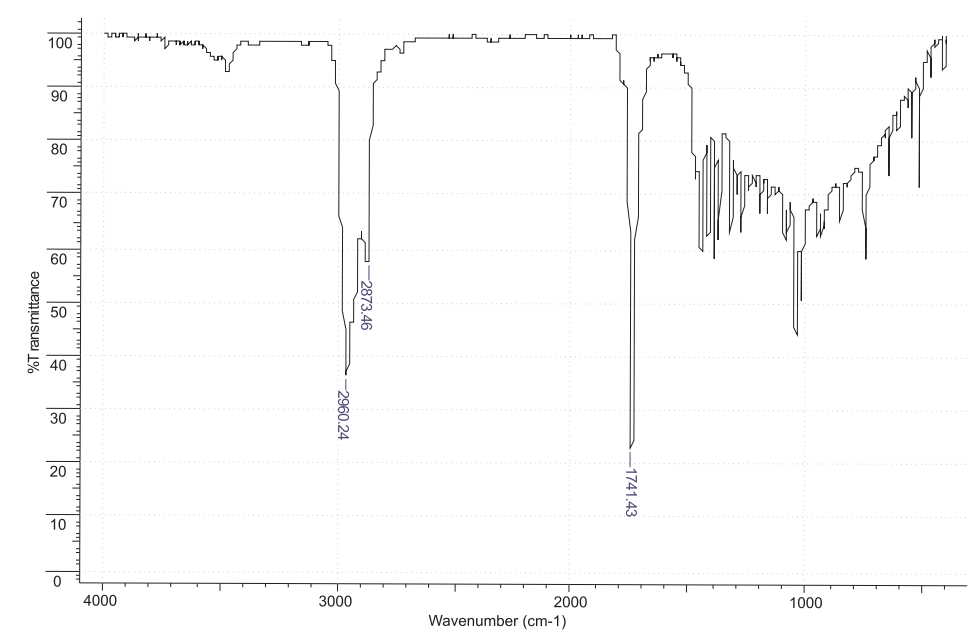

Figura 1S. Espectro IV da cânfora

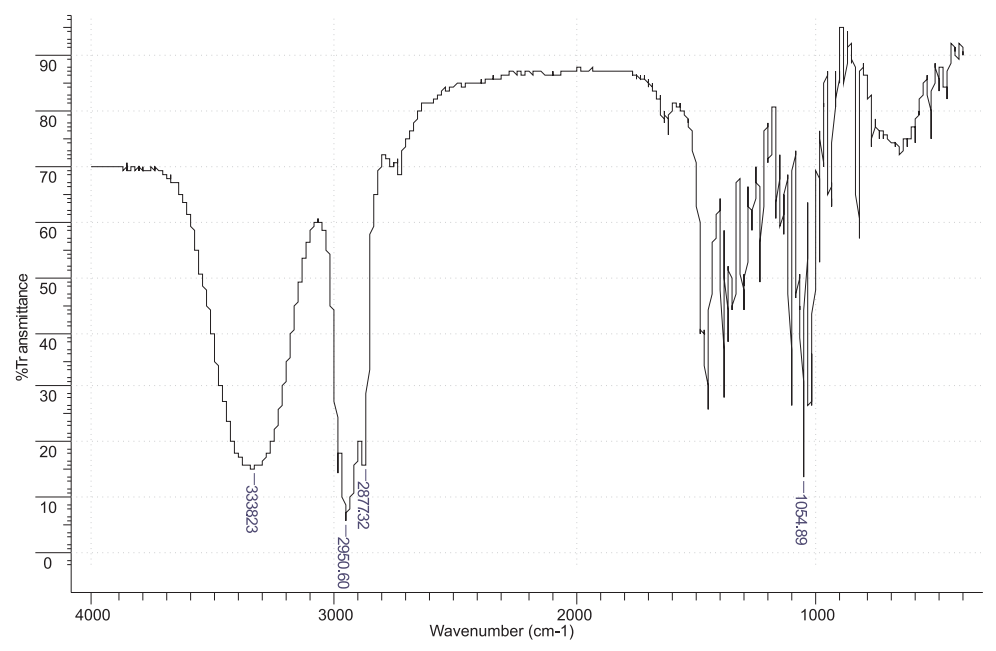

Figura 2S. Espectro IV do borneol

*e-mail: anabernardo@iq.ufrj.br 


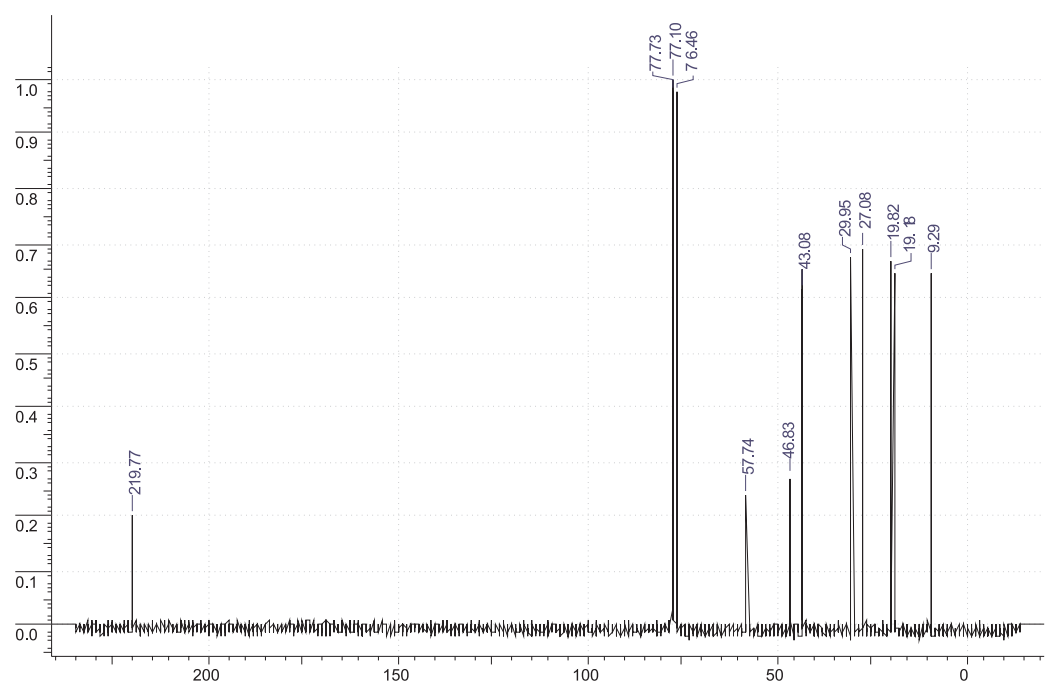

Figura 3S. Espectro RMN ${ }^{13} \mathrm{C}$ da cânfora

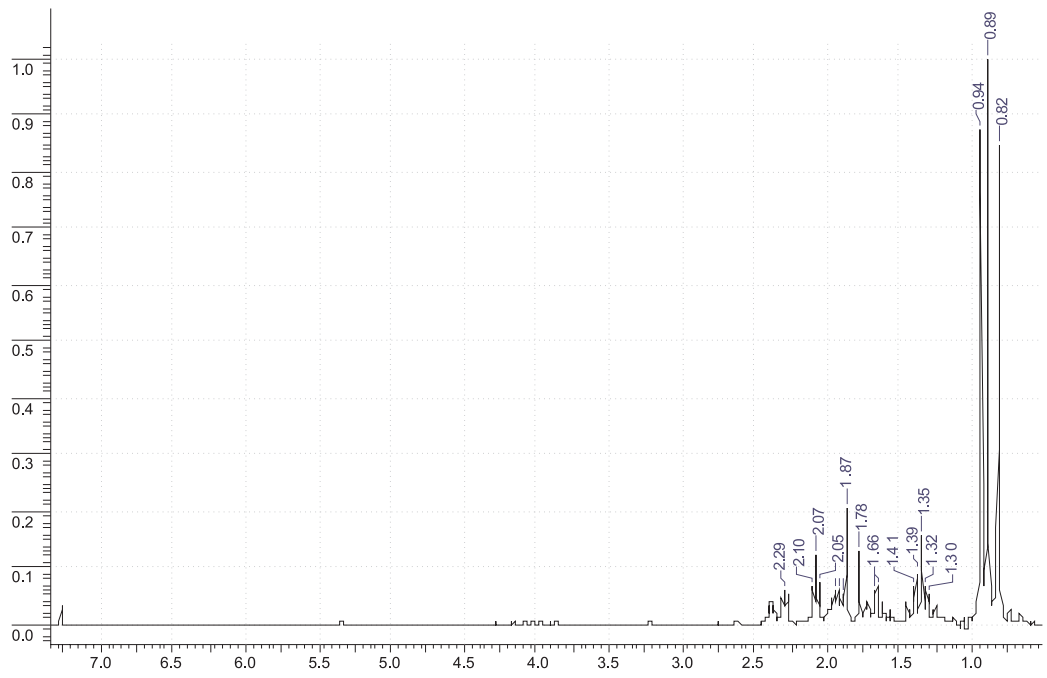

Figura 4S. Espectro RMN de ${ }^{l} \mathrm{H}$ da cânfora

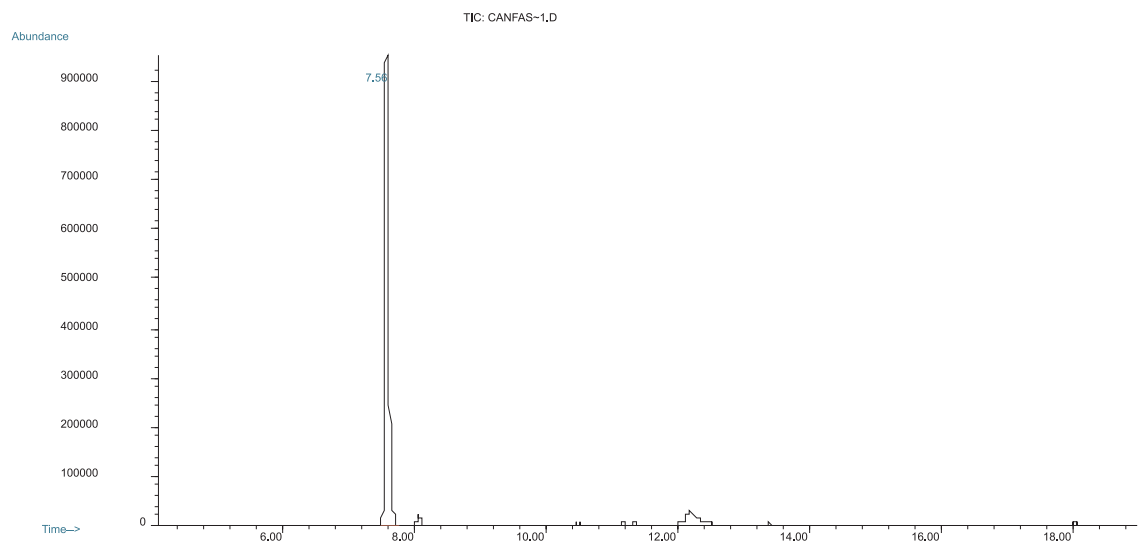

Figura 5S. Cromatograma CG da cânfora 